Meta

Journal des traducteurs

Translators' Journal

\title{
La stylistique collective dans la formation linguistique et professionnelle des traducteurs et interprètes de conférence
}

\section{Pietro Intravavaia et Pierre Scavee}

Volume 39, numéro 1, mars 1994

La traduction et l'interprétation dans la Belgique multilingue

URI : https://id.erudit.org/iderudit/003224ar

DOI : https://doi.org/10.7202/003224ar

Aller au sommaire du numéro

Éditeur(s)

Les Presses de l'Université de Montréal

ISSN

0026-0452 (imprimé)

1492-1421 (numérique)

Découvrir la revue

Citer cet article

Intravavaia, P. \& Scavee, P. (1994). La stylistique collective dans la formation linguistique et professionnelle des traducteurs et interprètes de conférence. Meta, 39(1), 34-46. https://doi.org/10.7202/003224ar 


\title{
LA STYLISTIQUE COLLECTIVE DANS LA FORMATION LINGUISTIQUE ET PROFESSIONNELLE DES TRADUCTEURS ET INTERPRÈTES DE CONFÉRENCE
}

\author{
Pietro InTRaVala et PierRe SCAVEe \\ École d'interprètes internationaux, \\ Université de Mons-Hainaut, Mons, Belgique
}

\section{INTRODUCTION}

Notre propos dans ces pages est de livrer quelques réflexions qui nous viennent d'une longue expérience en matière de formation linguistique et professionnelle de traducteurs/interprètes à l'École d'Interprètes Internationaux (Université de Mons-Hainaut). Nos réflexions s'organisent aussi autour d'un ouvrage publié il y a quelques années et auquel nous faisons référence car les principes que nous nous apprêtons à développer s'y trouvent déjà exposés : il s'agit d'un Traité de stylistique comparée, qui est pour l'essentiel une recherche fondamentale dont les retombées pédagogiques ont contribué à faire évoluer la problématique de l'enseignement des langues en général et de la traduction/interprétation en particulier.

Ce sont, au départ, des raisons essentiellement pragmatiques qui ont présidé à la mise en place de nos théories stylistiques et à l'élaboration des ensembles didactiques qui en ont découlé. Il s'agissait au départ de former, souvent à partir de débutants complets, des spécialistes du multilinguisme capables de traiter un assez grand nombre de thèmes relativement spécialisés de manière à pouvoir en débattre avec quelque autorité, un sens affiné des pouvoirs multiples du langage, une perception aiguë des nuances et une parfaite authenticité d'expression dans la pratique active des langues étrangères. C'est sur cette authenticité que nous projetions d'enraciner par la suite toute la formation professionnelle des traducteurs / interprètes.

La tâche nous était facilitée par le fait que nous étions assurés de la validité des hypothèses structuro-globales et verbo-tonales héritées de l'École de St-Cloud-Zagreb qui, dès la création de l'EII, avaient constitué l'assise théorique et méthodologique des cours de langue des deux premières années. Cette philosophie de l'enseignement des langues avait exercé sur l'équipe montoise une profonde influence lorsque nous avions dû par la suite assurer la formation professionnelle des traducteurs /interprètes.

Ceci nous permet au passage de dire tout ce dont l'institution est redevable à Raymond Renard, fondateur de l'EII qui fut l'un des premiers à pressentir la fécondité du structuro-globalisme. Toute la réputation de notre Institut est fondée sur cette orientation saine de l'enseignement des langues qu'il a su lui imprimer dès sa création.

Il ne saurait être question d'approfondir ici les principes théoriques et méthodologiques qui sous-tendent cette philosophie particulière de l'enseignement des langues et leur connexion avec la méthodologie de l'interprétation. Nous renvoyons le lecteur intéressé à la bibliographie que nous présentons en annexe. Disons simplement, pour la compréhension 
des motivations qui sont à l'origine de notre recherche de stylistique comparée que l'enseignement/apprentissage de la langue étrangère à l'EII était marqué par une insistance particulière sur la langue parlée, la discrimination auditive, la correction phonétique, une approche inductive, non intellectualisée et non contrastive de la grammaire, l'acquisition simultanée et solidaire des micro-compétences (audio-phonatoire, rythmicomélodique, phonostylistique, kinésique, proxémique, etc.), la consubstantialité situation/ discours permettant de gérer équitablement tant les conventions énonciatives et interactionnelles constitutives de la communication que les règles linguistiques formelles.

Dès lors, les candidats traducteurs-interprètes formés à cette école pouvaient se prévaloir d'une connaissance affinée des langues étrangères, connaissance acquise sous la guidance de pédagogues-phonéticiens qui, en mettant une insistance au départ à restructurer la perception auditive et à peaufiner la prononciation de l'apprenant, ouvraient la voie à la maîtrise de la langue étrangère dans toute la plénitude de ses composantes linguistiques et communicatives.

Et pourtant, on sentait confusément qu'il manquait aux étudiants arrivés au seuil de la $3^{\mathrm{e}}$ année un «je ne sais quoi» qui eût conféré à leur expression une authenticité totale. Notre expérience de professeurs de langue nous amenait à constater que les productions de nos étudiants, comparées à celles de l'autochtone exprimant, dans une situation analogue, les mêmes intentions de communication étaient davantage marquées par les spécificités psychologiques et dialectiques de leur langue maternelle que par des particularités phonétiques, prosodiques, lexicales ou morpho-syntaxiques. Pour être acceptables, elles n'en étaient pas moins inappropriés, car elles dérogeaient aux normes qui régissent le mode de sentir et d'exprimer propre à la communauté dont on apprend la langue. Autrement dit, il était peu probable que, placé dans une situation de communication analogue, l'autochtone aurait formulé la réalité suivant les mêmes schémas. Bref, nos étudiants francophones parlaient l'italien «à la française».

Ainsi une analyse stylistique attentive des conversations d'étudiants francophones apprenant l'italien laissait apparaître en transparence les schémas conceptuels et discursifs de leur langue maternelle : tantôt la réalité était présentée de manière abstraite et conceptuelle là où une formulation plus concrète et terre-à-terre eût été de rigueur; tantôt une présentation psychologique, synthétique, symbolique et subjective eût été préférable à une formulation logique, analytique, discursive et objective du réel. Aux antipodes, nous pouvions effectuer des observations analogues auprès de nos étudiants italophones venus des Écoles sœurs pour perfectionner leur français : l'amplitude affective (avec toutes ses implications sur les composantes kinésique et proxémique: ampleur gestuelle ponctuant le discours, réduction de la distance conversationnelle) était disproportionnée à la situation, l'exubérance sentimentale débordante ou du moins perçue comme telle par le francophone à qui le message était destiné. En un mot, le discours de nos italophones, surtout méridionaux, se signalait souvent par un baroquisme linguistique et communicatif hérité de leur langue maternelle et qui se déversait dans l'expression française.

Nous nous trouvions en terrain de connaissance. Les travaux de l'École phonologique de Prague nous avaient depuis longtemps familiarisés à l'existence d'un «crible» phonologique qui conditionne tous les ressortissants adultes d'une communauté linguistique à canaliser leur faculté auditive dans le sens des entités abstraites de leur code, les phonèmes, dont la distribution psychophonique détermine la perception et, partant, la reproduction des caractéristiques orales d'une nouvelle langue.

Notre travail de phonéticiens verbo-tonalistes, confrontés en permanence à l'apparition de systèmes d'erreurs quasiment identiques pour tous les ressortissants d'une même communauté et appelant des procédures de remédiation similaires, nous avaient amenés à étendre la notion de «crible» aux composantes suprasegmentales (rythmico-mélodique), 
tonémique (dans le cas des langues tonales), kinésique (système mimo-gestuel), proxémique (distance conversationnelle), autant de systèmes de référence liés de manière indissociable et qui constituent autant d'entraves à l'acquisition par l'apprenant d'une nouvelle langue.

L'enseignement de la langue italienne au niveau avancé nous amenait à nous imprégner progressivement de l'idée que les membres d'une même communauté sont prédéterminés dans l'apprentissage d'une autre langue par les «cribles» psychologique et dialectique de leur langue maternelle qui coiffent et englobent tous les éléments évoqués plus haut. Dès lors, l'individu qui apprend une langue étrangère, tout en développant une compétence linguistique satisfaisante, continue souvent d'actualiser les potentialités de la nouvelle langue suivant les schémas affectifs, intellectuels et discursifs de la langue maternelle.

Or, nous avions l'ambition d'amener les apprenants à s'exprimer dans la langue étrangère (en l'occurrence l'italien) avec la plus parfaite authenticité de telle sorte que la parole individuelle s'inscrive exactement dans les normes qui régissent un mode de sensibilité particulière ainsi que des approches dialectiques spécifiques.

Encore fallait-il définir la nature de cette sensibilité particulière, ce «je ne sais quoi» qui confère à l'italien sa spécificité propre.

À ce stade, l'accès à l'authenticité langagière est souvent livré au heureux hasard, à la rencontre occasionnelle, à la sensibilité linguistique de l'apprenant affinée par un séjour dans le pays ou à l'intuition créatrice du maître. Faute d'avoir mené à bien des recherches fondamentales de stylistique comparée pour différentes langues, l'enseignement au niveau terminal se ramène souvent à un bricolage empirique de matériaux hétéroclites et l'apprentissage à une navigation en solitaire au gré des lectures fortuites de textes authentiques qui rendent très aléatoire l'accès à la parfaite maîtrise de la langue étrangère.

C'est la négation de tout enseignement. Nous voulions aller plus loin et dépasser le stade de l'empirisme pédagogique.

Il nous fallait, avant de concevoir des méthodes d'enseignement, mener une recherche fondamentale pour déceler, analyser, répertorier les marques spécifiques de l'italianité et si possible les regrouper autour de polarisations affectives susceptibles d'ouvrir la voie à la spécificité psychologique collective de l'autre communauté.

$\hat{A}$ ce stade, nous voudrions souligner une divergence d'orientation fondamentale entre la recherche entreprise au Département d'italien de l'EII et celle de nos prédécesseurs; Vinay et Darbelnet (1963), Malblanc (1968). Tant chez Vinay et Darbelnet que chez Malblanc, on trouve indistinctement confondues une étude fort pertinente des mécanismes de traduction et une tentative - à la vérité fort prudente et limitée aux plus grandes généralités - de caractériser l'appréhension particulière du réel que reflètent les langues que l'on compare. C'est ainsi que l'on trouve dans ces ouvrages un diagnostic de base qui montre dans l'allemand et l'anglais deux langues à vocation réaliste (préférence pour le «plan du réel») et dans le français une langue à vocation conceptuelle (préférence pour le «plan de l'entendement»). Tant Malblanc que Vinay et Darbelnet ne vont guère au-delà de ce jugement interprétatif élémentaire, sans doute en raison d'une répugnance très compréhensible à paraître manier des concepts jugés «métaphysiques» ou «subjectifs» ou «impressionnistes» comme le «génie» des langues. Cependant, faute d'oser aborder une herméneutique psychologique des langues en présence, si aléatoire soit-elle, ces deux ouvrages perdent une bonne part de leur utilité pratique. Tout se passe comme si l'on se déchargeait sur l'aspirant traducteur ou interprète du soin de manier cette notion inconfortable de «génie» des langues.

En d'autres termes, l'apprenti traducteur ou interprète est forcé de s'en remettre à sa propre intuition, au sens empirique qu'il peut avoir de la sensibilité propre aux peuples 
dont il pratique la langue, avant de tenter d'appliquer les multiples mécanismes de traduction inventoriés par Malblanc, Vinay et Darbelnet, et ce n'est guère qu'après coup, la traduction faite, qu'il découvre un peu à la manière de $M$. Jourdain qu'il fait, sans le savoir, une «transposition», un «chassé-croisé» ou une «modulation»!

C'est pourquoi les recherches du Département d'italien, parce qu'elles s'assignent une fonction pédagogique, se sont voulues résolument «interprétatives», voire «psychologiques», au risque d'encourir les reproches faciles de ceux qui ne craignent rien tant que les idées simplement vraisemblables mais non rigoureusement démontrables.

\section{LA NOTION DE STYLE COLLECTIF}

Nous nous sommes donc mis à la recherche de l'authenticité de la langue italienne, c'est-à-dire, en dernière analyse, de sa structure psychologique.

Nous avions la certitude d'explorer un terrain en friche. La stylistique au sens où l'entendait Charles Bally, c'est-à-dire le réservoir des potentialités d'expression affective d'une langue, ne pouvait se trouver au centre des préoccupations de la stylistique comparée, du moins dans l'option qui est la nôtre. En effet, la confrontation après coup de deux stylistiques internes, la comparaison de deux inventaires de ressources et moyens étaient d'un intérêt assez mince. S'il peut être édifiant, à la rigueur, dans une optique de pure investigation linguistique, de dresser en parallèle des inventaires des moyens expressifs de langues structuralement différentes telles que le magyar et un dialecte amérindien, l'intérêt de ce genre de confrontation est absolument nul quand on compare des langues sœurs comme le français et l'italien, qui ne sont à tout prendre que des modalités quelque peu divergentes d'un même idiome néo-latin. La proximité entre les deux langues ne se marque pas seulement par une parenté structurale et lexicale aussi étroite que possible, mais aussi par un potentiel commun de virtualités expressives.

L'exemple le plus banal que l'on peut citer est sans doute la ressource de l'inversion affective (sujet - complément - verbe) au lieu de la séquence progressive (sujet verbe - complément). Le recours à l'une ou l'autre de ces démarches est une alternative théoriquement ouverte aussi bien au français qu'à l'italien, mais, alors que l'italien y recourt de façon usuelle, l'inversion n'est jamais en français contemporain qu'une tournure inhabituelle tant quantitativement (fréquence infiniment moins grande) que qualitativement (niveau de langue familier ou populaire). Tout aussi classique les virtualités de formation de substantifs, adjectifs ou adverbes dérivés (en -ismo;-istico; -mente) pour lesquels le français se trouve fréquemment devant une ressource potentielle analogue à l'italien, si ce n'est que l'usage se montre à cet endroit singulièrement plus réticent. En d'autres termes, les ressources potentielles d'expressions affectives sont pratiquement identiques dans les deux langues. Il n'y a rien ou presque dans l'une dont on ne puisse trouver un équivalent formel dans l'autre, si ce n'est que l'utilisation qui en est faite est sensiblement différente. Autrement dit, le clavier est identique, c'est la manière dont on en joue qui diffère.

Nous disposions également d'un autre concept de référence: le style individuel, c'est-à-dire la marque individuelle d'une parole. Pas plus que la stylistique, le style individuel ne nous était d'aucune utilité, car nous ne pouvions songer à enseigner la langue de tous au travers d'écritures qui sont l'œuvre de talents particuliers. Inversement les sublimations esthétiques ou communicatives ne peuvent se goûter que par comparaison, par contraste.

Or, une étude comparative des langues comme celle que nous avons entreprise nous a amenés à postuler l'existence, entre ces deux extrêmes du kaléidoscope stylistique, d'une instance linguistique intermédiaire entre les notions saussuriennes de langue et de parole qui est le comportement collectif d'une communauté linguistique : un style collectif. 
Nous avons donc mis en évidence, à partir de centaines de marques stylistiques provenant des horizons les plus divers de la langue que, au delà des caractères individuels (spécificités régionales, sous-codes socioculturels, variantes d'âge et de sexe), tous les ressortissants d'une communauté linguistique ont en commun de ressentir, structurer et exprimer le réel d'après des schémas affectifs, intellectuels et dialectiques identiques, suivant des rituels interactionnels non conscients, hérités de la tradition culturelle qui a modelé les sensibilités collectives.

En résumé, si la stylistique au sens que nous avons indiqué désigne le réservoir des possibilités d'expressions affectives d'une langue et le style individuel le choix préférentiel d'une personne puisant dans ce potentiel linguistique, le style collectif concerne le choix préférentiel propre à toute une collectivité qui, parmi toutes les possibilités d'expressions affectives, privilégie certaines d'entre elles selon un mode de sensibilité particulier. Celui-ci se manifeste par une certaine manière d'utiliser la gamme des expressions affectives que la langue met à la disposition de l'usage (stylistique). Cette manière, répétons-le, est commune à l'ensemble de la collectivité linguistique.

Dans notre tentative de capter, d'expliquer, de faire comprendre les polarisations affectives de la langue italienne saisie dans l'usage collectif qui en est fait, nous avons inventorié un certain nombre de complexes affectifs qui sont autant de constellations d'affects, de sentiments, de valeurs morales, esthétiques et culturelles, de structurations des rapports entre le monde sensible et la pensée formant des touts organiques animés d'une cohésion interne. Ces complexes s'organisent en un ensemble, dessinent une forma mentis commune dont les caractères sont suffisamment affinés pour qu'il soit possible de dresser une typologie psychologique collective dont le comportement linguistique est le reflet.

Nous livrons ici les conclusions auxquelles ces recherches sur le style collectif italien ont abouti :

1. L'italien se situe de préférence sur le «plan du réel», alors que le français s'abstrait plutôt sur le «plan de l'entendement». C'est ce que l'on peut déduire d'usages idiomatiques ou de manières de dire qui sont parfaitement libres, en ce sens qu'on pourrait chaque fois leur opposer d'autres possibilités d'expression: l'usage d'adverbes déictiques, la banalisation des verbes, l'usage de mots passe-partout comme cosa. Cette proximité à l'égard du réel sensible se retrouve dans l'usage de certains verbes auxiliaires ou d'une concordance des temps particulière.

2. Ce réalisme italien est essentiellement un réalisme psychologique : l'originalité de l'italien tient à la forte charge passionnelle que contient cette proximité sensorielle, à l'ampleur des réactions que provoque le monde sensible. Nous avons détecté le «taux de réactivité» élevé de l'italien dans des marques institutionnalisées par la langue : diminutifs, augmentatifs, laudatifs, péjoratifs, etc. Nous avons été amenés à opposer des concepts, comme psychologique et logique, subjectif et objectif, sensoriel et intellectuel, synthétique et analytique, symbolique et discursif pour caractériser les styles collectifs italien et français (voir l'usage en italien du datif éthique, de l'inversion syntaxique, des tournures elliptiques, l'extrême ductilité syntaxique...). Nous avons poussé la vérification de notre diagnostic au niveau dialectique en suivant le mouvement de la pensée dans ses méandres et replis: crescendo, rectification concessive, ajustement à la pensée et au sentiment de l'interlocuteur, appel au bon sens et à l'évidence, etc.

3. À cette tendance énoncée sub 1 - et nuancée sub 2 - dans laquelle on peut reconnaître la vocation fondamentale de l'italien, il convient d'opposer une tendance antinomique faite d'un goût immodéré de l'italien pour l'abstraction conceptuelle, tendance qui apparaît comme une superstructure, comme un héritage culturel contingent. 
4. Ces tendances contradictoires s'organisent de façon contrastive - structurale, par conséquent - ce qui confère à l'italien une note de baroquisme, par comparaison au français, plus sobre, plus «classique».

En prenant comme il se doit cum grano salis des désignations plaisantes qui sont à tout prendre des appellations assez suggestives des comportements que nous décrivons, on peut finalement reconnaître un certain nombre de complexes affectifs sur lesquels se polarise la sensibilité commune des personnes ressortissant à cette tradition linguistique :

- un "complesso di San Francesco" $(F)$ par lequel il faut entendre toutes les manifestations d'une sensibilité participationniste à base d'humanisme chrétien: compréhension et compassion universelle, pathétique et sensiblerie pouvant aller jusqu'au populisme et au misérabilisme mais aussi élans de spontanéité, immédiateté d'appréhension du beau comme du laid, de la joie comme de la souffrance...

- un «complesso di Pietro Bembo» $(B)$ qui recouvre un goût hédoniste d'origine essentiellement culturelle et savante pour la cadence et le rythme des phrases, le cursus sonore, la redondance, l'ampollosità et l'anfrattuosità des périodes...

- un «complesso di Benedetto Croce» $(C)$ déjà évoqué précédemment, à savoir un penchant immodéré pour les dérivations abstraites, pour la virtuosité mentale et les jeux du conceptualisme philosophique, tendance évidemment culturelle et académique qui touche au paroxysme caricatural dans le jargon des hommes politiques, mais qui aboutit aussi à une mode généralisée par l'influence de la presse écrite et parlée...

I un complexe mineur que l'on propose d'appeler "complesso del meccanico» $(M)$, se rapportant plus probablement à une tendance populaire, cas particulier du réalisme fondamental de l'italien; il s'agit d'un ensemble de marques stylistiques reflétant l'ingéniosité traditionnelle du peuple italien qui le porte à saisir la réalité comme un organisme, une mécanique, un système qu'il faut faire fonctionner (thème de l'embốtement, de l'articulation, du système ou du remède à appliquer, etc.);

w enfin on peut reconnaître une curieuse hybridation de ce dernier complexe avec celui de Croce (MC), sorte de faux concret créant une illusion de profondeur, un pseudo-philosophisme qui recouvre bien souvent une pensée assez inconsistante.

Nous regrettons évidemment de devoir exposer ici de façon aussi sommaire et de présenter à la manière de postulats cette théorie des complexes affectifs, mais ce n'est pas le lieu d'en démontrer la validité au moyen de nombreux exemples qui pourraient l'étayer.

Précisons que ces marques stylistiques se rencontrent à tous les niveaux, c'est-à-dire qu'elles peuvent être données par des éléments lexicaux ou des expressions toutes faites formant lexème, tantôt pour l'emploi de tournures grammaticales particulières, tantôt par la structuration de la pensée dans un raisonnement plus ou moins complexe, sans compter la composante phonostylistique relevant des éléments prosodiques, kinésiques, proxémiques, etc.

En l'occurrence, la classification linguistique des faits stylistiques importe peu: ce qui importe, c'est l'unité d'effet produit, ce qu'on pourrait nommer à la manière de Léo Spitzer un «étymon spirituel» qui ici ne concerne pas une «parole» (ceuvre littéraire), mais un usage généralisé (style collectif italien) et que la théorie à laquelle nous nous référons propose d'appeler un «complexe affectif».

Qu'il nous suffise d'illustrer par deux exemples la vocation fondamentale de l'italien au réalisme psychologique.

Le premier énoncé contient un certain nombre de marques stylistiques convergentes relevant essentiellement de la grammaire et pour lequel l'usager dispose d'une latitude totale dans le choix que lui offre la langue. 
Il piccolo imprenditore si teneva carissimi gli apprendisti che lavoravano bene. Guai a chi glieli toccava. Quando gliene moriva o gliene andava via uno prendeva il lutto.
Le patron d'une petite entreprise était aux petits soins pour les apprentis qui travaillaient correctement. Il ne faisait pas bon toucher à un cheveu de leur tête. Quand l'un d'entre eux mourait ou quittait l'entreprise, c'était un drame.

Le réalisme est attesté par la présence d'un verbe très général accompagné d'un déictique andar via ainsi que le raccourci elliptique de si teneva carissimi donné par l'emploi d'un adjectif en lieu et place d'un adverbe qui exprime une proximité plus grande à l'endroit du réel tangible que ne le ferait l'adverbe, plus abstrait, plus conceptualisé. On reconnaîtra également dans le recours à la formation elliptique une propension à l'appréhension synthétique de la réalité. Ce réalisme est psychologique en ce qu'il se colore de subjectivité dans l'usage en cascade des pronoms réfléchis dans si teneva carissimi, guai à chi glieli toccava, quando gliene moriva uno, gliene andava via uno. L'approche de la réalité en italien est différente, plus subjective, en l'occurrence plus participative, en ceci qu'elle fait référence explicite à la personne qui est au centre de l'action ou en subit les effets. Le procédé apparaît comme le reflet d'une structure mentale qui organise la réalité autour de la personne impliquée émotivement. La tonalité générale de la phrase italienne dénote une participation affective et une charge émotionnelle plus forte qu'en français. Même le banal bene, dont la possibilité de traduction littérale risque de masquer la spécificité italienne, connote une facette particulière de l'esprit «franciscain» que nous décrivions plus haut: la simplicité du code d'estimation qui ordonne les choses, les faits et les gens autour de notions immédiates comme le beau, le bien, le juste. La vision italienne du monde repose sur un code d'évaluation morale binaire dont les composantes sont des valeurs élémentaires jugées évidentes pour la conscience de chacun comme le couple bene/male, giusto/sbagliato, bello/brutto, etc.

Le deuxième exemple nous conduit sur les territoires qui marquent la limite de notre domaine d'investigation, au niveau de l'organisation générale du discours, dans des contextes où la liberté de réalisation est totale. À ce niveau, nous parlerions volontiers de «dialectique comparée», mais il est évident qu'on y retrouve des tendances collectives, une mode italienne bien reconnaissable, en l'occurrence, le réalisme et la force de l'évidence. Ce peut être, par exemple, l'organisation implicite des liens de causalité selon une juxtaposition binaire. C'est un type d'exposition fort typique qui consiste à enfermer l'interlocuteur dans un discours dichotomique, à reconstituer pièce par pièce le raisonnement supposé d'une personne afin de rendre sensible une argumentation par l'évocation d'une saynète.

Campons le décor: chez les Rossi, la nuit a été particulièrement agitée. Pierino s'est glissé sous le lit et est resté accroché aux ressorts du sommier. Après des tentatives infructueuses pour le tirer de ce mauvais pas, le père se résigne à appeler un médecin de garde. Pour emporter l'adhésion du conjoint qui se montre réfractaire à ce genre de solution, Monsieur Rossi développe ce type d'argumentation:

La società mi mette o no mi mette a disposizione una guardia medica? Si. Questa guardia medica ha il dovere di mandarmi gratis un dottore per assistermi? Si. E allora, perchè non dovrei approfittarne anche per venire a capo dei capricci di questo piccolo scocciatore ? Gli altri si arrangino.
À partir du moment où la société met à ma disposition un service de garde dont le devoir est de m'envoyer gratuitement un médecin pour m'assister, je ne vois pas pourquoi je n'en profiterais pas pour venir à bout des caprices de ce sale moutard. Que les autres se débrouillent. 
Les valeurs qui sous-tendent cette façon provocatoire d'argumenter sont le réalisme et la force de l'évidence et confirment le diagnostic fondamental que nous avons porté : goût du concret qui pousse à reconstituer toutes les étapes du raisonnement, recherche de l'adhésion de son interlocuteur à qui l'on mime la scène pour souligner davantage les valeurs d'évidence et de bons sens du raisonnement. La transcription du discours est impuissante à recréer l'impression globale à laquelle contribuent tous les éléments extralinguistiques de la communication: il faudrait pouvoir transcrire les moyens non verbaux mis en œuvre pour établir, maintenir le contact et s'assurer que le message passe : l'insistance du regard, la mimo-gestuelle ponctuant tout le déploiement du rituel phatique, (expression faciale de celui qui sollicite et reconnaît implicitement chez son interlocuteur l'aptitude à se rendre à l'évidence); toute une rythmique gestuelle agissant sur le suprasegmental (variations de hauteurs ou d'intensité, amplitude sonore, étirement expressif des syllabes); rétrécissement de la distance conversationnelle et contact physique caractéristique des gens du Sud. De plus, la traduction littérale risque parfois de voiler la spécificité italienne, mais pas au point de ne pas faire sentir combien il est improbable que des circonstances analogues amènent spontanément des expositions semblables en français. La surenchère affective et le concrétisme anecdotique sonnent étrangement lorsqu'on essaie de les transposer en français : ce sont ces éléments qui confèrent si souvent aux discours italiens l'impression de truculence qu'ils créent chez l'observateur francophone à qui ils rappellent, non sans raison, les manières d'être de leurs propres méridionaux, l'emphase méditerranéenne qui a fait le succès des pièces de Marcel Pagnol.

Nous croyons en avoir assez dit pour illustrer cette instance linguistique intermédiaire entre la langue et la parole. Nous proposons une analogie vestimentaire qui achèvera de mettre en place nos catégories dans leurs rapports respectifs.

La stylistique, suivant cette comparaison, correspondrait à l'étoffe, au tissu et aux ressources techniques du tissu pour une part, et pour une autre part, aux conventions sociales comme pourraient l'être le port de la cravate ou du faux col; poussant jusqu'au bout cette comparaison, on trouverait dans le choix d'accepter ou de rejeter ces conventions quelque chose d'analogue à la hiérarchie sociale des différents niveaux de langue, plus ou moins «nobles», plus ou moins «populaires». Le style collectif, lui, correspondrait à cette réalité indiscutable qu'il existe une «coupe italienne» et une mode acceptée assez généralement qui régit le choix des coloris et des contrastes plus ou moins voyants. Quant au style personnel, il serait l'affirmation d'une élégance singulière qui pourrait être, selon les cas, un refus des canons vestimentaires, (quelque chose comme l'affectation d'un style «old fashion»), bien plus souvent une exagération des tendances collectives, (le «dandysme»: on suit la mode dans ses dernières outrances, on la devance même), enfin, tout à fait exceptionnellement, une recherche d'excentricité totale, à vrai dire aussi rare en matière de langage que dans l'analogie que nous proposons: dans un cas les poètes, dans l'autre les hippies!

Si l'on admet qu'il existe une approche particulière de la réalité qui est commune à l'ensemble d'une collectivité linguistique et qui se reflète dans l'usage stylistique, bref un style collectif qui est une actualisation du potentiel de ressources d'expression affective (stylistique) sans que l'on puisse cependant le reconduire à un parti pris individuel de réaliser une expression originale (style personnel), on conviendra que cette entité linguistique constitue l'élément central des recherches de stylistique comparée et que son acquisition cognitive et pratique est l'objet par excellence de ce que nous avons nommé déjà «stylistique comparée appliquée», qu'il s'agisse de la formation professionnelle ou de l'enseignement de la langue étrangère aux traducteurs / interprètes. 


\section{STYLISTIQUE COMPARÉE APPLIQUÉE}

Considérons d'abord le cas des traducteurs et interprètes.

Nous ne songeons pas à nier que le problème de traduction soit à chaque fois un cas d'espèce posé par une parole singulière qui est l'expression orginale d'une pensée et d'intentions strictement individuelles, mais il est bien évident que l'évaluation de ce style personnel ne peut se faire qu'à partir d'un paramètre ou étalon qui est la mode collective. Nous l'avons dit, le style individuel n'est jamais qu'exceptionnellement une originalité intégrale, une création ex nihilo (poésie surréaliste, par exemple); il est soit une rupture, une distanciation à l'égard des modes collectives, — assez rarement, d'ailleurs — soit, le plus souvent, une intensification des caractères de ces modes. Dans les deux cas, les marques originales ne peuvent être appréciées que par rapport aux usages collectifs dont elles sont des variants, et la faute de traduction consisterait précisément à rapporter au style de l'individu et à une intention personnelle ce qui n'est que l'expression banalisée d'une sensibilité collective. Bien plus : dans l'acte de traduction, c'est-à-dire du transfert d'une langue à une autre des paroles individuelles, l'écart relatif de la parole individuelle par rapport à l'usage ou à la mode qu'est le style collectif doit être reporté, aussi fidèlement que possible, à partir du style collectif de la langue-cible, lui-même variant par rapport à celui de la langue de départ. Imaginons le cas concret déjà évoqué par Devoto d'un traducteur appelé à transposer en anglais une page de D'Annunzio. On conçoit que le baroquisme qui caractérise le style personnel de cet auteur demande à être apprécié par rapport à une norme stylistique collective de l'italien, elle-même substantiellement plus baroque que la norme anglaise; le report de l'écart individuel devra, par conséquent, se faire en tenant compte des écarts existant entre les styles collectifs italien et anglais, faute de quoi l'impact provoqué par la page traduite sur le lecteur britannique s'en trouverait indûment exagéré.

Bien plus couramment encore que dans ces cas, somme toute marginaux, que sont les traductions littéraires, les documents fonctionnels qui représentent la plus grosse partie du matériel que les traducteurs sont appelés à traiter dans l'exercice de leur profession réclament en toute priorité une sensibilisation aux usages du style collectif. Que de fois, en effet, des traducteurs inexpérimentés ne s'évertuent-ils pas à transférer dans la langue d'arrivée des marques affectives qu'ils attribuent indûment à un souci de style personnel inexistant alors qu'elles ne sont, en tout état de cause, que le reflet d'une manière collective de sentir et d'exprimer qui est celle de la langue de départ et qui en aucun cas ne demande à être déversée dans la langue-cible!

On voit par conséquent que c'est l'affinement de la sensibilité aux règles qui régissent le style collectif des langues en présence qui constitue la pierre de touche dont le traducteur se sert pour apprécier exactement les textes qu'il est appelé à traiter; c'est le seul moyen qui lui permettra d'atteindre la véritable «congruence», notion que d'aucuns ont voulu opposer, non sans quelque raison, à la notion dangereuse de «fidélité à tout prix» mais qui n'est, à nos yeux, que la véritable, l'authentique fidélité au texte, c'est-à-dire celle qui tient compte comme il se doit des modalités différentes de sentir et d'exprimer dans les différentes langues et se garde notamment de rapporter à une intention individuelle ce qui n'est qu'une spécificité collective.

Imaginons, pour illustrer ce qui risquerait de paraître des théorisations bien abstraites, la phrase suivante rencontrée sous la plume d'un journaliste qui, à bien des égards, mérite d'être considéré comme un des plus purs reflets de l'italianité : «Le forze di polizia hanno consentito al governo dei colonelli di galleggiare nella provvisorietà».

Le contraste patent du concret et de l'abstrait nous livre un étymon de baroquisme. Cette notion de baroquisme nous est en quelque sorte donnée dans l'absolu puisque nous pouvons y voir, par l'accolement d'expressions antithétiques abstraites et concrètes, une 
recherche outrancière de l'effet qui est bien le propre de ce qu'il est convenu de qualifier de baroque. Mais s’il est vrai que la notion de baroque est nécessairement une notion relative qui implique que l'on puisse lui opposer une norme plus sobre - «classique»-, l'exercice de transposition en français nous confirme la justesse de notre diagnostic. Il est patent que la phrase proposée est strictement intraduisible littéralement en français: «les forces de police ont permis au gouvernement des colonels de se maintenir à flot dans la provisoireté». Plus encore que l'inexistence en français d'un substantif équivalent à l'abstrait provvisorietà, le contraste heurté du concret et de l'abstrait invitera sans doute le traducteur à traduire simplement: ... se maintenir provisoirement, atténuant à la fois l'expression concrète (en renonçant à la métaphore) et l'expression abstraite (par le recours à l'adverbe, plus «qualifiant» donc moins abstrait que le substantif dérivé, d'ailleurs impossible). Certes, on ne laissera pas d'éprouver le sentiment d'une légère perte dans la traduction: si le sens strictement dénotatif a été effectivement rendu, il ne reste pas moins que la phrase italienne évoque de façon plus expressive l'idée de précarité et d'instabilité, à la fois par la métaphore et le substantif dérivé (substantif «d'état» invitant par conséquent à une appréhension plus subjective que l'adverbe français proposé comme équivalent). Aussi pourrait-on imaginer que, par souci d'exhaustivité de la traduction, on propose des traductions un peu plus explicites, soit par exemple: se maintenir provisoirement tant bien que mal (en recourant au procédé de l'étai), ou encore (en gardant l'expression métaphorique devenue en français un simple cliché et en ne lui opposant aucune expression abstraite contrastive) : se maintenir à flot provisoirement. On voit que, dans tous les cas, un tel souci de rendre à tout prix la plus grande expressivité italienne entraînerait une lourdeur de la phrase française, ce qui ne peut que nous confirmer dans notre diagnostic, à savoir la préférence du français pour l'expression plus sobre, plus strictement énonciative, plus classique, par conséquent.

Il va de soi que toute notre argumentation porterait à faux si l'on était en droit de supposer que la phrase italienne relève d'un baroquisme qui serait propre à un auteur déterminé, qu'elle soit une marque de «style individuel» et non le reflet d'un «style collectif». Or, on peut tout au plus admettre chez l'auteur de cette phrase une légère part d'intention «volontaire et consciente» (marque du «style» selon Bally), qui est ici une acceptation plutôt qu'une recherche délibérée de l'effet (on vérifie par là ce que nous avons dit précédemment du «style personnel» en tant qu'exagération plus ou moins caractérisée des tendances collectives de la communauté linguistique). En fait, une phrase comme celle-là, qui se signale à notre attention par l'effet légèrement grossissant que la part de style personnel apporte aux tendances du style collectif, en évoque pour nous une infinité d'autres : elle rappelle à notre mémoire une foule d'expressions analogues dans lesquelles aucune intention particulière ne peut être suspectée, qui ne sont que l'expression la plus naturelle et la plus spontanée chez le sujet parlant italien.

Citons pour exemple ceci, qui est bien la façon de s'exprimer la plus normale, la plus banale, la plus usuelle en italien: «I recenti provvedimenti hanno riportato le cose alla normalità». On peut, à propos de cette phrase absolument dénuée de toute intention d'auteur, faire le même raisonnement qu'à partir de la précédente : le contraste structural d'un concret (cose) et d'un abstrait (normalità) permet d'induire un étymon de baroquisme, un peu moins flagrant, certes, si on ne l'envisage que d'une manière absolue, mais non moins patent si on lui oppose une traduction en une langue à vocation classique comme le français. En effet, ici encore, toute traduction littérale s'avère impossible («ramener les choses à la normalité»). C'est également la même démarche de traduction que nous évoquions pour l'exemple précédent qui va nous suggérer la traduction la plus authentiquement française: cette fois aussi, il s'agira d'atténuer les effets de contraste en conceptualisant davantage le premier élément (cose) et en descendant d'un cran l'abstraction du 
second (normalità). On s'achemine ainsi vers une traduction «normaliser la situation» dans laquelle le recours au verbe (plus immédiat, donc plus concret que le substantif) et le remplacement d'un substantif au sens banal et immédiat par un autre plus conceptuel viendront atténuer l'amplitude du contraste baroque.

L'intérêt utilitaire de ce jeu de combinaisons alternées n'apparaît que si le traducteur a été sensibilisé au préalable au style collectif de l'italien, plus baroque que le français et l'erreur du traducteur non averti consisterait à attribuer à une intention personnelle ce qui n'est que l'expression la plus banalisée d'une sensibilité collective.

On peut faire le même raisonnement à partir de l'exemple: «l'europeismo è andato indietro» qui devient «l'idée européenne a regressé». À le décrire du seul point de vue des procédés techniques, on ferait état de transpositions diverses. Mais l'orientation est donnée à la traduction par tout autre chose : la nécessité consciemment perçue d'atténuer encore une fois le contraste entre abstrait et concret pour donner au français le ton homogène qu'il affectionne. Dès lors, les mutations des catégories grammaticales sont suggérées: atténuer d'un côté l'abstraction en traduisant «europeismo» par «idée européenne» et estomper le concret «andare indietro» par un verbe plus abstrait «régresser», tout cela de manière à obtenir la syntonie recherchée.

C'est à ce moment que la connaissance claire et consciente des mécanismes révèle son utilité, quand elle est précédée d'une connaissance claire et consciente de ce qu'exige l'adaptation d'un style collectif à un autre.

Nous disons aussi: une attitude générale à l'égard du texte. C'est ce que nous appelons la congruence. Le mot n'est pas nouveau et sert parfois à couvrir une conception de la traduction, notamment des textes littéraires, que nous sommes loin de partager. Une assez bonne définition serait celle-ci : dans le passage d'une langue à une autre, c'est l'ajustement scrupuleux de l'énoncé linguistique à la situation qui l'accompagne, compte tenu des différences socioculturelles des peuples qui s'expriment dans ces langues. Pour nous, cela revient à dire : veiller toujours à ce que l'énoncé linguistique dans la langue d'arrivée soit bien la façon naturelle dont les usagers de cette langue s'exprimeraient dans la circonstance envisagée. Nous considérons que cette attitude a une valeur universelle, mais, ce dont nous sommes parfaitement assurés, c'est qu'elle a une particulière importance pour des traductions d'italien en français ou de français en italien. Il n'est pas exagéré de dire que quatre-vingt pour cent de ce qui se dit ou s'écrit dans l'une de ces langues supporterait à la rigueur la traduction littérale, sans offenser les règles et les usages, si ce n'est que, dans bien de ces cas, la tournure ainsi obtenue n'aurait aucune chance d'être celle que produirait spontanément l'usager. Sensibiliser le traducteur à la manière d'éprouver et d'énoncer qui est propre aux langues qu'il traite, c'est la tâche la plus fondamentale de la «stylistique comparée appliquée à la traduction».

Toutes les considérations pratiques auxquelles nous nous sommes livrés jusqu'ici à propos des traducteurs s'appliquent a fortiori aux interprètes de conférences. La véritable fidélité à la pensée de l'orateur implique que l'interprète s'efforce de transposer le message au public à qui il est destiné dans le plus grand respect des spécificités stylistiques et argumentatives qui sont celles de la communauté à laquelle celui-ci appartient. Souvent, certaines interprétations - surtout simultanées - sont totalement incompréhensibles au consommateur pur parce que l'interprète s'obstine à ne voir dans le message à délivrer qu'un simple transcodage de formes linguistiques. Ce risque d'interférences est surtout fréquent dans l'interprétation simultanée de deux langues romanes. Ce que l'on appelle habituellement d'un terme très général le «recul», la distanciation par rapport à l'orateur doit être le fruit d'une double opération mentale qui consiste d'abord à déverbaliser les messages en s'affranchissant de l'emprise de la forme pour opérer une reformulation 
congruente en langue d'arrivée qui tienne compte des modulations requises par la sensibilité collective de la communauté d'appartenance du public concerné.

Quelques lignes suffiront pour évoquer le rôle de la stylistique collective dans la formation linguistique des traducteurs / interprètes. La parfaite authenticité qu'on escompte d'eux passe inévitablement par une sensibilisation d'ordre intellectuel et pratique à cette instance linguistique intermédiaire entre la langue et la parole que nous nommons style collectif, dont les marques constituent le matériau didactique que l'on rencontre inévitablement sur son chemin au cours d'un enseignement avancé ou terminal de la langue étrangère.

C'est la condition sine qua non de toute rencontre psychologique, intellectuelle et affective entre ressortissants de traditions socioculturelles différentes, c'est-à-dire de toute communication empathique. C'est grâce à la connaissance et à l'assimilation d'un code affectif propre à la collectivité socioculturelle dont on apprend la langue que l'on acquiert l'aptitude à créer une perméation des idées et des sentiments, a créer les conditions qui assurent à notre discours les meilleures chances d'être reçu, reconnu et estimé parce que potentiellement consonant avec la sensibilité de notre interlocuteur telle qu'elle a été formée par une tradition culturelle séculaire. Condition indispensable de la compréhension entre les peuples, c'est l'objectif qu'il faut toujours avoir présent à l'esprit quant on fait métier d'enseigner les langues, a fortiori dans une école d'interprètes.

Certes, l'affinement de la sensibilité aux règles qui régissent le style collectif implique, comme nous l'avons dit, que soient menées à bien des recherches fondamentales en stylistique comparée pour différentes langues, et la méthodologie de la recherche que nous avons définie pourrait constituer une orientation pour toute personne désireuse d'explorer ce domaine en friche. Cette démarche consiste en une transposition au style collectif de la méthode du cercle philologique appliquée aux études de style par Léo Spitzer et corrigée, pour pallier certains risques de subjectivité, par la stylistique structurale de Riffaterre. Nous croyons que cette démarche est transposable sur une autre langue que l'italien, encore que le français, langue sobre et classique par excellence, ne puisse dévoiler sa spécificité que par des éclairages multiples à partir de plusieurs autres langues.

Malgré l'ampleur de la recherche, nous ne saurions trop engager les stylisticiens à perfectionner la démarche et à prospecter ce domaine aux retombées capitales pour la didactique des langues et la méthodologie de l'interprétation. Comprendre et faire comprendre, c'est aussi permettre d'assimiler. Nous pensons que l'acquisition d'une langue étrangère n'est complète que si l'on parvient à faire siennes les démarches mentales et affectives propres à la langue étudiée, sans quoi il ne peut y avoir de véritable communication. L'enjeu en effet est bien celui-là : un élargissement de la conscience et un enrichissement de la sensibilité qui rendent possible un authentique échange intellectuel et affectif entre les hommes. C'est alors que l'étude des langues débouche sur un véritable humanisme.

\section{RÉFÉRENCES}

En plus des grands classiques de la stylistique, nous n'indiquerons ici que les ouvrages qui ont particulièrement retenu notre attention. Pour la didactique des langues, nous citons deux synthèses et une bibliographie qui pourront éclairer le lecteur non spécialiste, ainsi que certains de nos articles qui concernent la stylistique comparée appliquée à l'enseignement.

\section{Stylistique}

BARTH, G. (1961): Recherches sur la fréquence et la valeur des parties du discours en français, en anglais et en espagnol, Paris.

BALLY, Ch. (1951): Traité de stylistique française, Paris, Klincksieck.

DEVOTO, G. (1950): Studi di stilistica, Firenze, Le Monnier.

DEVOTO, G. (1951) : I fondamenti della storia linguistica, Firenze, Sansoni. 
DEVOTO, G. (1962) : Nuovi studi di stilistica, Firenze, Le Monnier.

GUIRAUD, P. (1969) : Essais de stylistique, Paris, Klincksieck.

MALBLANC, A. (1968) : Stylistique comparée du français et de l'allemand, Paris, Didier.

RIFFATERRE, M. (1971) : Essais de stylistique structurale, Paris, Flammarion.

SCAVEE, P. et P. INTRAVAIA (1979) : Traité de stylistique comparée, analyse comparative de l'italien et du français, Paris, Didier Érudition, 225 p.

SPITZER, L. (1970) : Études de style, Paris, Gallimard, (voir notamment la préface de J. Starobinski).

VINAY, J.-P. et J. DARBELNET (1963) : Stylistique comparée du français et de l'anglais, Paris, Didier.

\section{Didactique des langues}

GUBERINA, P. (1965) : «La méthode audio-visuelle structuro-globale», Revue de phonétique appliquée, 1, pp. 35-64.

INTRAVAIA, P. (1972) : «Situations, structures et explications», Revue de phonétique appliquée, 21, pp. 43-49.

INTRAVAIA, P. (1972) : «Quelques observations sur le déroulement des premières leçons dans la méthodologie SGAV», Bulletin de l'A.T.A.V., 6, pp. 1-29.

INTRAVAIA, P. et P. SCAVEE (1983): «Pour une méthodologie de l'enseignement de la traduction et de l'interprétation de conférences», Vingt ans d'enseignement et de recherche en traduction et en interprétation de conférence, Université de Mons-Hainaut, pp. 9-22.

INTRAVAIA, P. et P. SCAVEE (1984) : «Stilistica comparata e insegnamento della lingua italiana», L'insegnamento dell' Italiano in Belgio, Vrije Universiteit Brussel, pp. 51-61.

INTRAVAIA, P. et P. SCAVEE (1987): «Stylistique comparée, pluriculturalisme et évolution de la problématique SGAV», Revue de phonétique appliquée, 82-83-84, pp. 161-166.

INTRAVAIA, P. et P. SCAVEE (1989): «Empathie et congruence, une approche communicative nouvelle en didactique des langues», Travaux de didactique du FLE, Université Paul Valéry, Montpellier II, 22, pp. 57-76.

JIMENEZ, P. (1977): "Contribution à une bibliographie sur la méthodologie SGAV et le système verbotonale», Revue de Phonétique Appliquée, 41, pp. 81-93.

LADERCHI, E. (1973-1974) : Scenarii Stilistici, mémoire de licence sous la direction de P. Scavée et P. Intravaia, École d'Interprètes Internationaux de l'Université de Mons.

RENARD, R. (1976) : La méthodologie SGAV d'enseignement des langues. Une problématique de l'apprentissage de la parole, Paris, Didier.

SCAVEE, P. (1977) : «L'application des principes de la méthodologie audio-visuelle et structuro-globale à la préparation professionnelle des interprètes de conférence», Bulletin de l'A.T.A.V., Mons, 4, pp. 21-25.

SCAVEE, P. (1972) : «Après l'audio-visuel», Revue de phonétique appliquée, 21, pp. 77-83.

SCAVEE, P. et P. INTRAVAIA (1975) : «Stylistique comparée et enseignement SGAV», Revue de phonétique appliquée, 35, pp. 119-148.

SCAVEE, P. et P. INTRAVAIA (1976) : «La stylistique comparée dans l'enseignement SGAV du deuxième niveau», Actes du Ve colloque international sur la méthodologie structuro-globale audio-visuelle, Revue de phonétique appliquée, 39-40, pp. 287-290. 